\title{
Filtering and packaging data: The problem of representation
}

\author{
MARTHA KARREB $Æ K$
}

This article discusses some of the challenges involved in textual representation of audio-visual data for discourse and micro-analytic purposes. Today it is more important than ever to pay critical attention to this issue due to the widespread, but often relatively untheorized, use of video data in Conversation Analysis and related fields in the social and human sciences. The article presents a critical discussion of the relationship between audio-visual data, the researcher's impression of these data, and the textual representation generated through representation practices. The discussion highlights the challenges involved in representation of spoken language and of other types of (non-verbal) information, as well as the complications that emerge when researcher and research participants have different linguistic and/or cultural backgrounds (using research on children as an example). The article argues that representation of research data constitutes an analytic step in the research process, and that it therefore needs serious and critical reflection. In addition, it is argued that the representation is not just an analytic tool but also a way for an intended audience to gain insight into the analytic process. These different functions need to be carefully balanced and considered when transforming audio-visual data into textual representations. 